\title{
Antiviral Activity of Gemcitabine Against Echovirus 30 Infection in Vitro
}

\author{
Hwa-Jung Choi ${ }^{1}$, Jae-Hyoung Song ${ }^{2}$, Kyungah Yoon ${ }^{3 *}$ \\ 1Department of Beauty Science, Kwangju Women's University, 165 Sanjeong-dong, Gwangsan-gu, \\ Gwangju 62396, Korea \\ 2Laboratory of Microbiology and Immunology, College of Pharmacy, Kangwon National University, \\ Chuncheon 24341, Korea \\ ${ }^{3}$ Department of Clinical Pathology, Daejeon Health Institute of Technology, Daejeon 34504, Korea
}

\author{
Corresponding \\ Kyungah Yoon PhD \\ Department of Clinical Pathology, Daejeon \\ Health Institute of Technology, \\ Daejeon 34504, Korea \\ Phone : +82-42-670-9166 \\ Fax : +82-42-670-9582 \\ E-mail : kayun@hit.ac.kr
}

Received : October 22, 2019

Revised : November 19, 2019

Accepted : December 3, 2019

No potential conflict of interest relevant to this article was reported.

Copyright (C) 2019 Journal of Bacteriology and Virology

(C) This is an Open Access article distributed under the terms of the Creative Commons Attribution Non-Commercial License (http://creativecommons.org/ license/by-nc/3.0/)
Echovirus 30 is one of the major causes of meningitis in children and adults. The purpose of our current study was to investigate whether selected antiviral drugs could provide antiviral activity against echovirus 30. Using RD cells, we assessed the cytopathic effect of echovirus 30, including viral RNA levels as indicators of viral replication. The effects of gemcitabine were compared to rupintrivir, a well-known antiviral drug. To understand the activity gemcitabine exerts on the viral life cycle, time course and time-of-addition assays were implemented. The most effective compounds against echovirus 30 were gemcitabine and rupintrivir, as demonstrated by their concentration-dependent activity. Gemcitabine affects the early stages of echovirus 30 infection by disrupting viral replication. However, gemcitabine failed to directly inactivate echovirus 30 particles or impede viral uptake into the RD cells. Gemcitabine can be considered as a lead candidate in the development of echovirus 30 antiviral drugs, specifically in the early stages of echovirus 30 replication.

Key Words: Echovirus 30, Gemcitabine, Early stages

\section{INTRODUCTION}

Human enteroviruses (EVs) are positive-sense, single-stranded, RNA viruses and belong to Picornaviridae (1). Sequence analysis of human enteroviruses further grouped them into four species (EV-A to $D$ ). Among these, six serotypes of EV-A (CAV2, 4, 6, 10, 16 and EV71) and four serotypes of EV-B (E6, 9, 30 and CAV9) have been detected in young children with sepsis-like illness across Edinburgh and the US (2-4).

Echovirus 30 (E30) is a key pathogen related with aseptic meningitis in humans (5, 6). It is highly prevalent on a global scale (7-9). The transmission routes of E30 may be direct such as fecal-oral and respiratory routes or indirect, such as via contaminated water (10).

Primary infection with E30 leads to replication of the virus in the tissue around the gastrointestinal tract, with transient viremia following (11). Infectious diseases caused by pathogenic organisms such as viruses are still responsible for the majority 
of hospitalization and death worldwide. Although considerable advancement has been made by using various antibiotic agents, there remain big challenges in the effective treatment of various infections $(12,13)$. In addition to numerous side effects in the use of antiviral agents due to their effect on the whole body, the rapid emergence of drug resistance adds a serious problem to human health $(14,15)$. Moreover, new viruses are constantly emerging through evolution or other biological events, leading to a continuing challenge in the control and prevention of infectious diseases. Consequently, in addition to discovering novel antiviral drugs for infection control, new strategies need to be expanded to maximize efficacy of the existing available drugs (16).

Gemcitabine is widely utilized in the treatment of pancreatic, ovarian, and non-small cell lung cancers (17). To date, however, no reports have described the antiviral activity of gemcitabine against E30. In this study, we assess the antiviral activity of gemcitabine against E30, and further elucidate its mechanism of action.

\section{MATERIALS AND METHODS}

\section{Viruses and cell lines}

E30 (ATCC, VR-692) was purchased from American Type Culture Collection (Manassas, VA, USA) and grown at $37^{\circ} \mathrm{C}$ in RD cells (human rhabdomyosarcoma cell line). RD cells were cultured in minimal essential medium (MEM), supplemented with $10 \%$ fetal bovine serum (FBS) and 1\% antibiotic-antimycotic solution. MEM, FBS, trypsin-EDTA, and antibiotic-antimycotic solution were purchased from Gibco BRL (Invitrogen Life Technologies, Karlsruhe, Germany) and tissue culture plates were purchased from Falcon (BD Biosciences, San Jose, CA, USA).

\section{Antiviral activity assay}

Antiviral activity was determined by sulforhodamine B (SRB) staining using the cytopathic effect (CPE) reduction assay method, following viral infection (18). One day before infection, RD cells (at $2 \times 10^{4}$ cells/well) were seeded onto a 96-well plate (Falcon, BD biosciences, San Jose, CA, USA). The next day, culture medium was altered to $1 \%$ FBS and the virus suspension was added at a $50 \%$ tissue culture infective dose $\left(\mathrm{TCID}_{50}\right)$. An appropriate concentration of the gemcitabine was added to the plate which was then cultured at $37^{\circ} \mathrm{C}$ in a $\mathrm{CO}_{2}$ incubator for 2 days until the appropriate $\mathrm{CPE}$ was generated. To fix cells, ice-cold $70 \%$ acetone was added to the plate. After $30 \mathrm{~min}$, the fixed cells were stained using SRB solution $(0.4 \%$ $(\mathrm{w} / \mathrm{v})$ in $1 \%$ acetic acid solution. To solubilize bound SRB, $10 \mathrm{mM}$ unbuffered Tris base solution was added to the plate. The absorbance was measured at $562 \mathrm{~nm}$ by a SpectraMax i3 microplate reader (Molecular Devices, Palo Alto, CA, USA) with a reference absorbance at $650 \mathrm{~nm}$. Cell viability (\%) was calculated for comparison, based on the optical density measured. In addition, images of SRB-stained cells were recorded using ImmunoSpot (CTL, ShakerHeights, OH, USA).

To test the effect of gemcitabine on the infectivity of E30 particles, E30 was pre-incubated with gemcitabine or rupintrivir at $4^{\circ} \mathrm{C}$. After $1 \mathrm{~h}, \mathrm{RD}$ cells were treated with pretreated or untreated $\mathrm{E} 30$ at $37^{\circ} \mathrm{C}$ and following a further $1 \mathrm{~h}$, unbound virus was eliminated. After washing using 2x PBS, RD cells were incubated in medium provided with or without gemcitabine or rupintrivir at $37^{\circ} \mathrm{C}$. Antiviral activity was measured using the SRB assay 2 days following (19).

\section{Time course experiment}

RD cells infected with TCID 50 E30 were harvested at various time points, including 4, 6, 8, 10, and 12 h post-infection, after which gemcitabine and rupintrivir were added. Total RNA was isolated the indicated time points post-infection. The levels of E30 RNA were analyzed using real time-PCR. 


\section{Time-of-addition (TOA) assay}

Gemcitabine and rupintrivir were then added onto the cells at $10 \mu \mathrm{M}$ or $2 \mu \mathrm{M}$, respectively, either before $(-1 \mathrm{~h})$, during $(0 \mathrm{~h})$, or after (1, 2, 4, 6, 8, 10 and $12 \mathrm{~h})$ E30 infection. After $14 \mathrm{~h}$, real-time (RT-) PCR analysis was conducted using Thunderbird SYBR aPCR Mix (Toyobo, Osaka, Japan).

\section{Real-time PCR}

Total RNA was extracted using the QIAamp viral RNA Mini kit (Qiagen, Valencia, CA, USA). The reverse transcription reaction was generated using an RNA $20 \mu \mathrm{l}$ reaction volume for 60 min at $42^{\circ} \mathrm{C}$, containing RNase inhibitor, murine Maloney leukemia virus reverse transcriptase with $5 \times$ buffer, oligo(dT) 15 primer and a dNTP mixture (all from Promega, Madison, WI, USA) according to the manufacturer's instructions. Quantitative RT-PCR analysis was conducted and cDNA was amplified using the CFX96 optical reaction real-time PCR system (Bio-Rad, Hercules, CA, USA) with Thunderbird SYBR qPCR Mix (Toyobo, Osaka, Japan). The following primers were used: E30 5'-NCR-sense, 5'-TCC TCC GGC CCC TGA ATG-3' and 5'-NCRantisense, 5'-GAA ACA CGG ACA CCC AAA G-3' (20).

\section{RESULTS}

\section{In vitro antiviral activity of gemcitabine against E30 infection}

We recently reported that gemcitabine has antiviral activity against human rhinoviruses (21). Here we confirmed the antiviral effect of gemcitabine on E30 by assessing the virus-induced cytopathic effect in RD cells using an SRB assay. E30 induced approximately $90 \%$ cell death in RD cells, and treatment of cells with $2 \mu \mathrm{M}$ of gemcitabine significantly increased cell viability up to $80 \%$. Furthermore, $10 \mu \mathrm{M}$ of gemcitabine fully preserved RD cells from cytotoxicity induced by E30 infection. Rupintrivir (positive control) exhibited strong antiviral activity against E30 up to a concentration of $0.016 \mu \mathrm{M}$ (Fig. 1A). We also assessed the cytotoxicity of gemcitabine and rupintrivir against RD cells. Gemcitabine and rupintrivir did not show cytotoxicity at $50 \mu \mathrm{M}$ and $2 \mu \mathrm{M}$ respectively (Fig. 1B). Collectively, these results suggest that gemcitabine possesses antiviral activity against $\mathrm{E} 30$ in vitro.
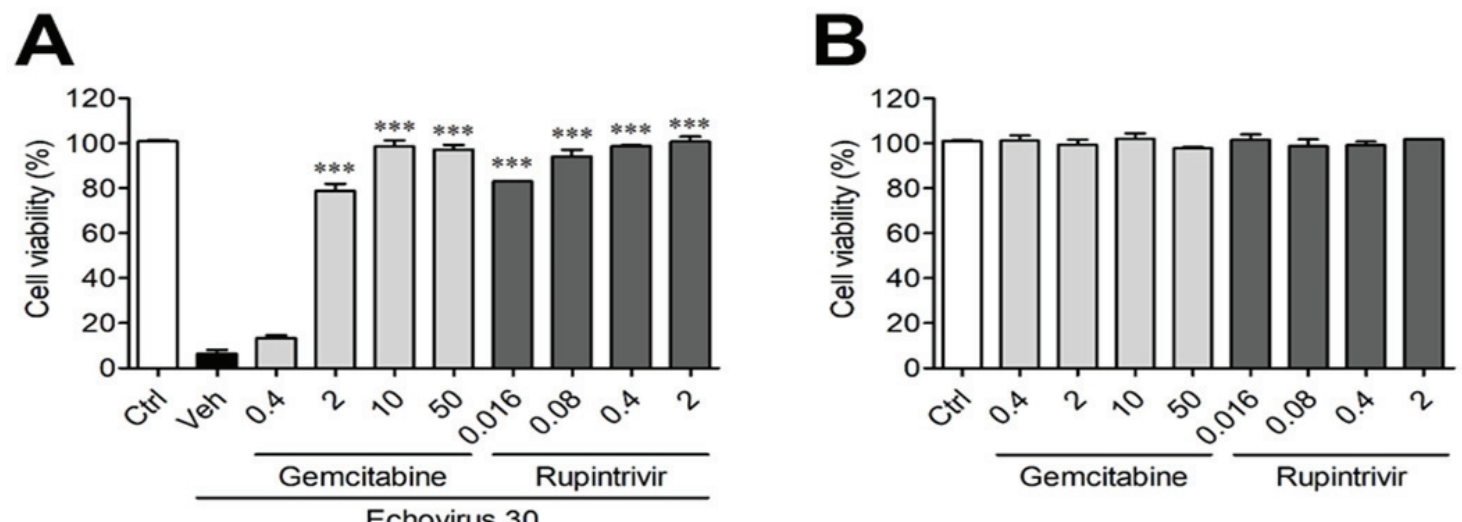

Fig. 1. Gemcitabine-mediated antiviral activity against E30 in vitro. (A) RD cells were infected with the TCID 50 (50\% cell culture infective dose) of E30, and treated with indicated concentrations of gemcitabine and rupintrivir. Cell viability was evaluated using an SRB assay, and results were determined based on absorbance at 524nm. Bar graphs show mean \pm SD. Control (Ctrl) was neither infected nor treated, while vehicle (Veh) was infected with E30 but not treated with gemcitabine and rupintrivir. (B) The cytotoxicity of gemcitabine was evaluated using an SRB assay in RD cells. ${ }^{* * *} P<0.001$, based on ANOVA with Bonferroni's multiple comparison test. 


\section{Gemcitabine affects intracellular processes during early stages of E30 infection}

Based on the results produced from the E30 in vitro assay, we conducted time course experiments to analyze the mechanism of action of gemcitabine. Viral RNA from E30 was detected using RT-PCR at various times points $(4,6,8,10$, and $12 \mathrm{~h}$ post-infection) and after gemcitabine $(10 \mu \mathrm{M})$ and rupintrivir $(2 \mu \mathrm{M})$ treatment of E30-infected cells. We could find E30 viral RNA as early as $8 \mathrm{~h}$ post-infection. Interestingly, gemcitabine was seen to block the proliferation of E30 in resemblance to rupintrivir (inhibitor of picornavirus $3 \mathrm{C}$ protease, Fig. 2). Based on the results of the time course experiments, we hypothesized that the antiviral effect of gemcitabine occurs relatively early in the infection process. To observe which step is influenced by gemcitabine, we performed a time-of-addition experiment. Gemcitabine $(10 \mu \mathrm{M})$ and rupintrivir $(2 \mu \mathrm{M})$ were added to the culture medium at $-1,0,1,2,4,6,8,10$ and $12 \mathrm{~h}$ after virus infection. E30 RNA replication was analyzed at 14 h post-infection.

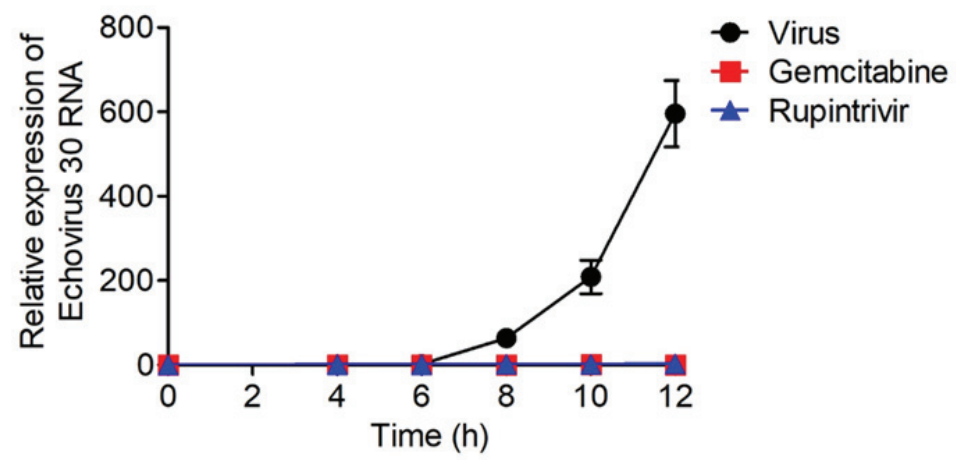

Fig. 2. Time course of $E 30$ infection. RD cells infected with the $T C I D_{50}$ of $E 30$ were harvested at the indicated time points after addition of $10 \mu \mathrm{M}$ gemcitabine, or $2 \mu \mathrm{M}$ rupintrivir (post-infection). Total RNA was isolated and E30 RNA was analyzed using RT-qPCR. Real-time PCR analyses were performed to determine the effect of gemcitabine, or rupintrivir on E30 NCR gene expression levels. Each value is the result of mean \pm S.D. of three independent experiments.

Treating cells with gemcitabine at $-1 \mathrm{~h}$ in respect to $\mathrm{E} 30$ infection did not inhibit the virus. However, gemcitabine treatment $10 \mathrm{~h}$ post-infection effectively suppresses E30 viral infection. In contrast, viral RNA somewhat increased with gemcitabine treatment at $12 \mathrm{~h}$ post-infection. This result suggests that gemcitabine did not inhibit E30 replication at an early state of infection, but instead, inhibits the stage of viral infection which occurs at $10 \mathrm{~h}$ or earlier post-infection (Fig. 3).

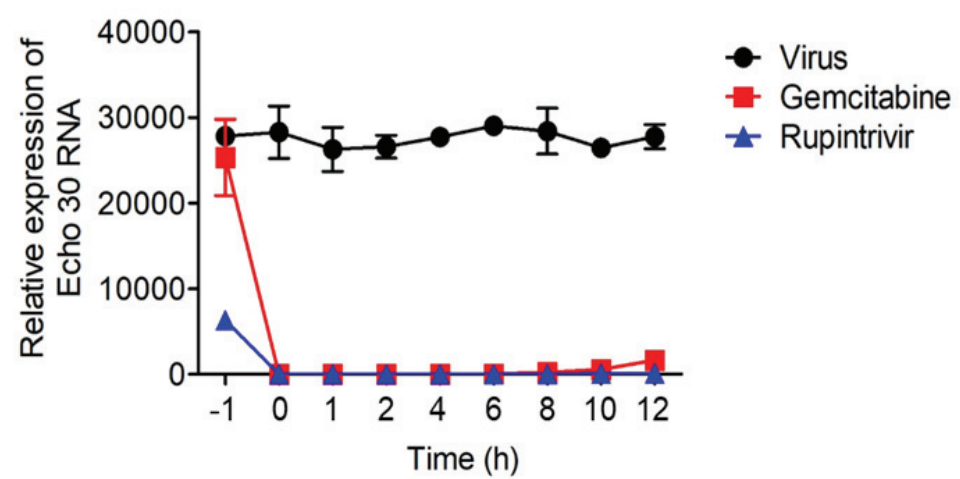

Fig. 3. Time-of-addition experiment to test effect of gemcitabine on the $\mathrm{E} 30$ viral cycle. Gemcitabine ( $10 \mu \mathrm{M})$, or rupintrivir (2 $\mu \mathrm{M}$ ) were added prior to, at the time of, or at indicated time points after viral infection of RD cells. The level of viral RNA expression was analyzed 14h post-infection. RD cells that were treated with drugs prior to viral infection were washed before infection. Each value is the result of mean \pm S.D. of three independent experiments. 


\section{The effects of gemcitabine on the infectivity of E30 particles}

The effects of gemcitabine on the infectivity of E30 particles were also investigated. The antiviral activity of E30 pre-incubated $(-1 \mathrm{~h})$ with gemcitabine and rupintrivir did not show significant results. In contrast, the sustained presence $(0 \mathrm{~h})$ of gemcitabine and rupintrivir during infection resulted in a marked increase in antiviral activity (Fig. 4). This result suggests that gemcitabine does not interact with E30 particles because pre-exposure of the virus to gemcitabine did not alter the infectivity of E30 particles.

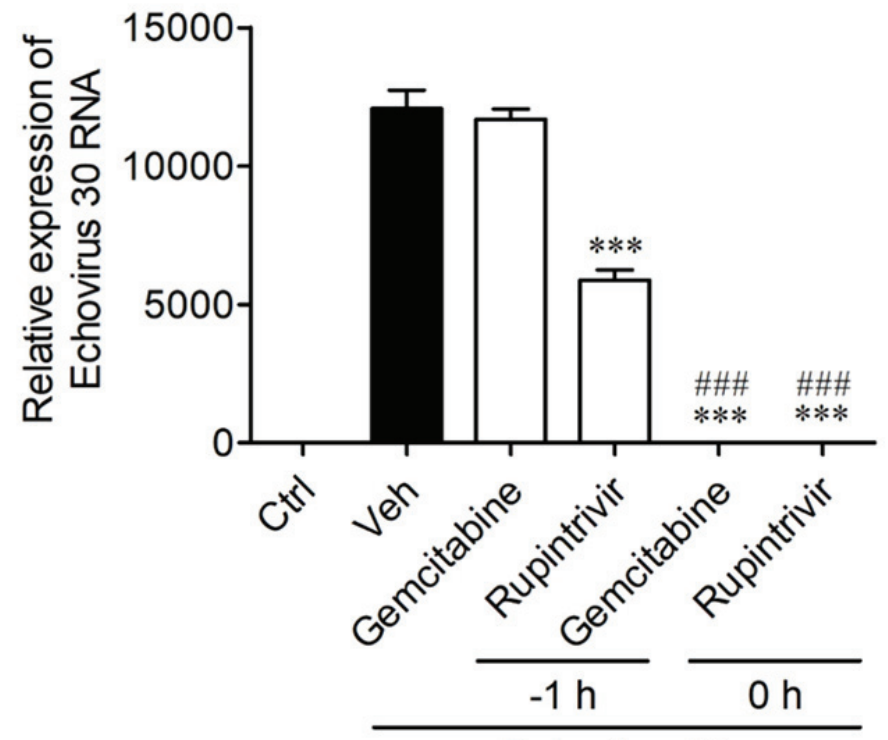

Echovirus 30

Fig. 4. Effects of gemcitabine on the infectivity of E30 particles. E30 particles were incubated with $10 \mu \mathrm{M}$ gemcitabine or 2 $\mu \mathrm{M}$ rupintrivir for $1 \mathrm{~h}$ at $4^{\circ} \mathrm{C}$. RD cells were then incubated with the treated or untreated virus for $1 \mathrm{~h}$ at $37^{\circ} \mathrm{C}$. Unbound virus was removed by extensive washing, and incubation was continued with or without $10 \mu \mathrm{M}$ gemcitabine or $2 \mu \mathrm{M}$ rupintrivir, at $37^{\circ} \mathrm{C}$. Antiviral activity was determined using RT-qPCR, $12 \mathrm{~h}$ post-infection. Preinc, pre-incubation of virus with the indicated drug without subsequent drug treatment of the infected cells; Inc., incubation of cells with the indicated drug after viral infection.

\section{NTPs alleviate the antiviral activity of gemcitabine}

Previously, it was reported that gemcitabine affects the intracellular levels of nucleotide triphosphate (NTP), especially decreasing CTP levels (22). Thus, we found that the addition of $50 \mu \mathrm{M}$ ATP, GTP, CTP and UTP significantly reduced the cell viability of RD cells treated with gemcitabine after infection with E30 (Fig. 5A and 5D). We found that the addition of pyrimidine nucleotides, UTP and CTP significantly reduced the antiviral activity of gemcitabine (Fig. 5D). Thus, the addition of gemcitabine limits UTP and CTP levels and may therefore restrict RNA synthesis (Fig. 5C and 5D). Collectively, we found that the anti-viral activity of gemcitabine may be controlled by reducing the levels of CTP and UTP after having been converted into gemcitabine triphosphate. 
A

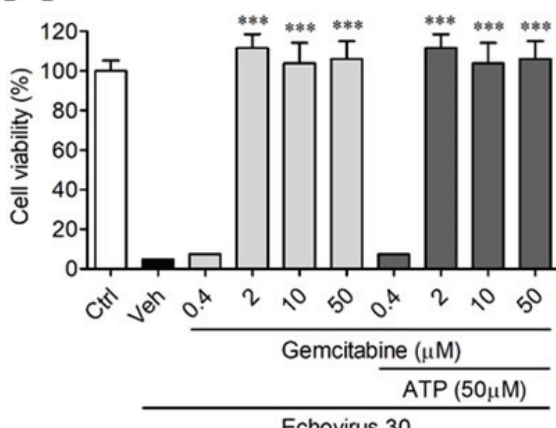

C

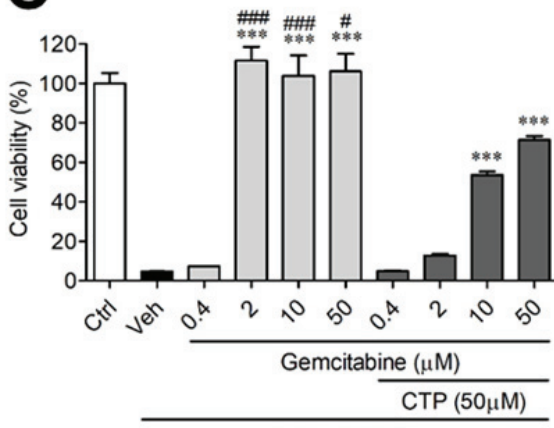

Echovirus 30
B

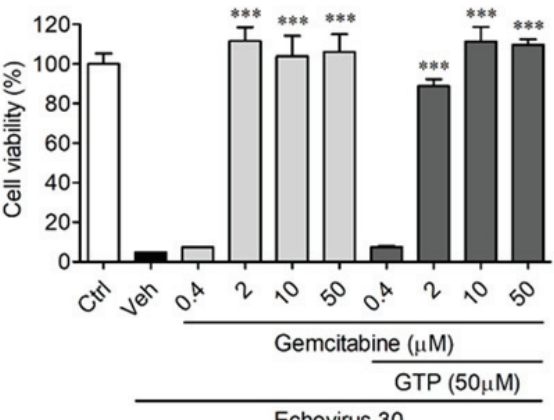

Echovirus 30

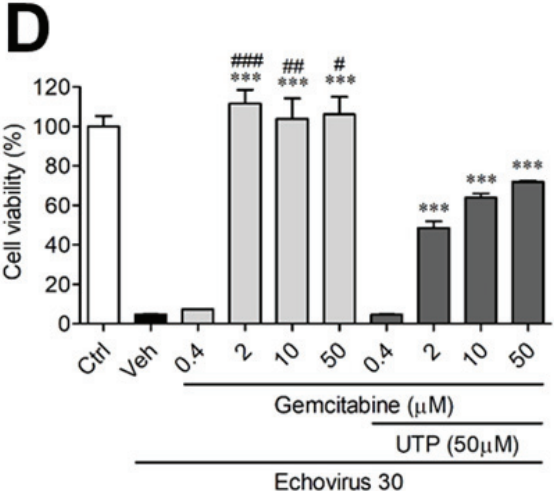

Fig. 5. NTP inhibits the antiviral activity of gemcitabine in vitro. E30-infected RD cells were incubated with gemcitabine for 4 $8 \mathrm{~h}$ at the indicated concentrations and (A) ATP, (B) GTP, (C) CTP, (D) UTP, and cell viability was determined. Data is expres sed as mean \pm SD of the cell viability percentage values obtained from three independent experiments carried out in a triplic ate. ${ }^{* * *} P<0.001$ for comparisons to the control, and ${ }^{\#} P<0.1,{ }^{\# \#} P<0.01$, ${ }^{\# \# \#} P<0.001$ for comparisons between NTP treatment and NTP non-treatment, and at the corresponding dose of gemcitabine.

\section{DISCUSSION}

Although vaccines are effective, immunity takes time to develop. Thus, they are of no use to the infected patient (23). Additionally, vaccines must be reformulated each year because of the antigenic drift and rendered ineffective when the formulation does not match the epidemic virus. For these reasons, some antivirals are sought to replace the vaccine by supplying long-continuous protection against both seasonal and pandemic viruses.

Gemcitabine is a nucleoside analog that inhibits cell growth by incorporating into DNA and preventing the action of DNA polymerases. It is approved for the treatment of multiple solid tumors (24). To date, however, the mechanism of action and effectiveness of gemcitabine against E30 have yet to be described. Therefore, we sought to examine whether gemcitabine possesses antiviral activity against E30 using RD cells. Gemcitabine did not show any cytotoxicity at concentrations of $0.4,2$, 10 and $50 \mu \mathrm{M}$. Treatment of cells with $10 \mu \mathrm{M}$ of gemcitabine significantly increased the cell viability to $100 \%$. Therefore, our results support the possibility of gemcitabine as a potential antiviral and therapeutic candidate.

Among the approaches used in determining the antiviral activity of gemcitabine, the time-of-addition assay identified inhibition of viral RNA replication as the mechanism of action. In our study, gemcitabine effectively inhibited the proliferation of echovirus 30, mirroring well-known rupintrivir, an inhibitor of picornavirus 3 C protease. Gemcitabine does not suppress the entry of the virus into the cell, but instead inhibits the stage of the viral life cycle that occurs no more than $10 \mathrm{~h}$ after 
infection. Therefore, we concluded that the antiviral effect of gemcitabine occurs relatively early during viral infection.

To realize the potential of gemcitabine as an antiviral candidate, its mechanism of activity must be understood. It is important to differentiate between viral inactivation (virucidal activity) from antiviral activity. Direct viral inactivation is an early event where the virus is inactivated before it infects the cells while antiviral activity involves killing the virus or the suppression of viral replication. It would be ideal for viral infection treatments to possess both virucidal and antiviral activities. In our study, gemcitabine did not directly interact with E30 particles as pre-exposure of the virus to gemcitabine did not alter the infectivity of E30 particles. Furthermore, addition of UTP and CTP significantly reduced the antiviral activity of gemcitabine by increasing the levels of pyrimidine nucleotides. The anti-echoviral activity of gemcitabine may be altered by reducing the levels of CTP and UTP after having being converted into gemcitabine triphosphate.

Thus, this study has revealed that gemcitabine could possess antiviral activity against E30. It does not directly inactivate E30 particles, but affects the initial stages of E30 infection by interfering with viral replication. The antiviral activity induced by gemcitabine treatment may be adjusted by reducing the levels of CTP and UTP after it has been converted into gemcitabine triphosphate. These results could be useful in the design of new inhibitors for use as anti-viral agents.

\section{CONFLICTS OF INTEREST}

There are no conflicts of interest relating to this article.

\section{ACKNOWLEDGMENTS}

This work was supported by a Daejeon Health Institute of Technology in 2016.

\section{REFERENCES}

1) Fieldhouse JK, Wang X, Mallinson KA, Tsao RW, Gray GC. A systematic review of evidence that enteroviruses may be zoonotic. Emerg Microbes Infect 2018;7:164.

2) Harvala H, Calvert J, Van Nguyen D, Clasper L, Gadsby N, Molyneaux P, et al. Comparison of diagnostic clinical samples and environmental sampling for enterovirus and parechovirus surveillance in Scotland, 2010 to 2012. Euro surveill $2014 ; 19$.

3) Maguire HC, Atkinson P, Sharland M, Bendig J. Enterovirus infections in England and Wales: laboratory surveillance data: 1975 to 1994. Commun Dis Public Health 1999:2:122-5.

4) Sharp J, Harrison CJ, Puckett K, Selvaraju SB, Penaranda S, Nix WA, et al. Characteristics of young infants in whom human parechovirus, enterovirus or neither were detected in cerebrospinal fluid during sepsis evaluations. Pediatr Infect Dis J 2013:32:213-6.

5) Peigue-Lafeuille $H$, Croquez N, Laurichesse $H$, Clavelou P, Aumaître O, Schmidt J, et al. Enterovirus meningitis in adults in 1999-2000 and evaluation of clinical management. J Med Virol 2002:67:47-53.

6) Amvrosieva TV, Titov LP, Mulders M, Hovi T, Dyakonova OV, Votyakov VI, et al. Viral water contamination as the cause of aseptic meningitis outbreak in Belarus. Cent Eur J Public Health 2001:9:154-7. 
7) Drebot MA, Nguan CY, Campbell JJ, Lee SH, Forward KR. Molecular epidemiology of enterovirus outbreaks in Canada during 1991-1992: identification of echovirus 30 and coxsackievirus B1 strains by amplicon sequencing. J Med Virol 1 994;44:340-7.

8) Lévêque N, Jacques J, Renois F, Antona D, Abely M, Chomel JJ, et al. Phylogenetic analysis of Echovirus 30 isolated during the 2005 outbreak in France reveals existence of multiple lineages and suggests frequent recombination events J Clin Virol 2010;48:137-41.

9) Khetsuriani N, Lamonte-Fowlkes A, Oberst S, Pallansch MA. Enterovirus surveillance--United States, 1970-2005. M M W R Surveill Summ 2006:55:1-20.

10) Palacios G, Casas I, Cisterna D, Trallero G, Tenorio A, Freire C. Molecular epidemiology of echovirus 30: temporal circulation and prevalence of single lineages. J Virol 2002;76:4940-9.

11) McWilliam Leitch EC, Bendig J, Cabrerizo M, Cardosa J, Hyypiä T, Ivanova OE, et al. Transmission networks and population turnover of echovirus 30. J Virol 2009;83:2109-18.

12) Metcalf CJE, Lessler J. Opportunities and challenges in modeling emerging infectious diseases. Science 2017;357: 149-52.

13) De Rycker M, Baragaña B, Duce SL, Gilbert $I H$. Challenges and recent progress in drug discovery for tropical diseases. Nature 2018;559:498-506.

14) Baker S, Thomson N, Weill FX, Holt KE. Genomic insights into the emergence and spread of antimicrobial-resistant bacterial pathogens. Science 2018;360:733-8.

15) Meylan S, Andrews IW, Collins JJ. Targeting Antibiotic Tolerance, Pathogen by Pathogen. Cell 2018;172:1228-38.

16) Willing BP, Russell SL, Finlay BB. Shifting the balance: antibiotic effects on host-microbiota mutualism. Nat Rev Microbiol 2011:9:233-43.

17) Baig J, Shokouh-Amiri M, Chan J, Chowdhery R, Danthurthy S, Venepalli NK. The Spectrum of Pulmonary Toxicity in Pancreatic Cancer Patients Receiving Gemcitabine Combination Chemotherapy. Case Rep Oncol 2019;12:506-12.

18) Song J, Yeo SG, Hong EH, Lee BR, Kim JW, Kim J, et al. Antiviral Activity of Hederasaponin B from Hedera helix against Enterovirus 71 Subgenotypes C3 and C4a. Biomol Ther 2014;22:41-6.

19) Choi HJ, Kim JH, Lee CH, Ahn YJ, Song JH, Baek SH, et al. Antiviral activity of quercetin 7-rhamnoside against porcine epidemic diarrhea virus. Antiviral Res 2009;81:77-81.

20) Kwon BE, Song JH, Song HH, Kang JW, Hwang SN, Rhee KJ, et al. Antiviral Activity of Oroxylin A against Coxsackievirus B3 Alleviates Virus-Induced Acute Pancreatic Damage in Mice. PloS one 2016:11:e0155784.

21) Song JH, Kim SR, Heo EY, Lee JY, Kim DE, Cho S, et al. Antiviral activity of gemcitabine against human rhinovirus in vitro and in vivo. Antiviral Res 2017:145:6-13.

22) Heinemann V, Schulz L, Issels RD, Plunkett W. Gemcitabine: a modulator of intracellular nucleotide and deoxynucleotide metabolism. Semin Oncol 1995:22:11-8. 
23) Nyanguile O. Peptide Antiviral Strategies as an Alternative to Treat Lower Respiratory Viral Infections. Front Immunol 2019;10:1366.

24) Cornacchia MA, Coromilas AJ, Gallitano SM, Frank RC, Halasz CL. Subacute cutaneous lupus erythematosus induced by gemcitabine in 2 patients with pancreatic cancer. JAAD Case Rep 2019:5:596-601. 